\title{
High-resolution QSM for functional and structural depiction of subthalamic nuclei in DBS presurgical mapping
}

\author{
Alexey V. Dimov, MS, ${ }^{1,2}$ Ajay Gupta, MD, ${ }^{2}$ Brian H. Kopell, MD, ${ }^{3}$ and Yi Wang, $\mathrm{PhD}^{1,2}$ \\ ${ }^{1}$ Meinig School of Biomedical Engineering, Cornell University, Ithaca; ${ }^{2 D e p a r t m e n t ~ o f ~ R a d i o l o g y, ~ W e i l l ~ M e d i c a l ~ C o l l e g e ~ o f ~ C o r n e l l ~}$ \\ University; and ${ }^{3}$ Department of Neurosurgery, Mount Sinai Health System, New York, New York
}

OBJECTIVE Faithful depiction of the subthalamic nucleus (STN) is critical for planning deep brain stimulation (DBS) surgery in patients with Parkinson's disease (PD). Quantitative susceptibility mapping (QSM) has been shown to be superior to traditional T2-weighted spin echo imaging (T2w). The aim of the study was to describe submillimeter QSM for preoperative imaging of the STN in planning of DBS.

METHODS Seven healthy volunteers were included in this study. T2w and QSM were obtained for all healthy volunteers, and images of different resolutions were reconstructed. Image quality and visibility of STN anatomical features were analyzed by a radiologist using a 5-point scale, and contrast properties of the STN and surrounding tissue were calculated. Additionally, data from 10 retrospectively and randomly selected PD patients who underwent 3-T MRI for DBS were analyzed for STN size and susceptibility gradient measurements.

RESULTS Higher contrast-to-noise ratio (CNR) values were observed in both high-resolution and low-resolution QSM images. Inter-resolution comparison demonstrated improvement in CNR for QSM, but not for T2w images. QSM provided higher inter-quadrant contrast ratios (CR) within the STN, and depicted a gradient in the distribution of susceptibility sources not visible in T2w images.

CONCLUSIONS For 3-T MRI, submillimeter QSM provides accurate delineation of the functional and anatomical STN features for DBS targeting.

https://thejns.org/doi/abs/10.3171/2018.3.JNS172145

KEYWORDS subthalamic nucleus; iron; deep brain stimulation; quantitative susceptibility mapping; electrode placement; functional neurosurgery; surgical technique

$\mathrm{T}$ HE subthalamic nucleus (STN) is a deep gray matter structure that is located in the midbrain and known to be involved in regulation of cognitive and motor functions. ${ }^{19,22}$ Due to the latter, the STN has been a primary target in deep brain stimulation (DBS) to improve parkinsonian symptoms. ${ }^{7,31,47}$ Success of DBS is critically dependent on accurate placement of the stimulation electrodes. ${ }^{48}$ However, precise targeting of the STN is a challenging task due to its small size, oblique orientation, and variations in anatomical location..$^{16,21,28,35}$ The STN is rich with iron that not only serves as a cofactor to generate glu- tamate for neurotransmission ${ }^{1,30}$ but also provides tissue contrast in MRI. ${ }^{45}$ Consequently, spin echo T2-weighted (T2w) sequence has traditionally been used for identification of the STN in presurgical DBS planning. ${ }^{6,16,23,26}$

Recently, gradient echo (GRE) sequences have been found to provide more sensitive contrast than $\mathrm{T} 2 \mathrm{w}$ sequences for depicting the STN. ${ }^{21}$ The GRE magnitude sensitizes iron with the T2* hypointensity and the GRE phase is proportional to the magnetic field generated by tissue iron. But both magnitude and phase signal at one location are affected by iron distribution in the surrounding

ABBREVIATIONS $\mathrm{AC}=$ anterior commissure; $\mathrm{AL}=$ anterolateral; $\mathrm{AM}=$ anteromedial; $\mathrm{CNR}=$ contrast-to-noise ratio; $\mathrm{CR}=$ contrast ratio; $\mathrm{DBS}=$ deep brain stimulation; $\mathrm{GPi}$ = globus pallidus internus; GRE = gradient echo; $\mathrm{MER}=$ microelectrode recording; $\mathrm{PC}=$ posterior commissure; $\mathrm{PD}=\mathrm{Parkinson}$ 's disease; $\mathrm{PL}=$ posterolateral; $\mathrm{PM}=$ posteromedial; QSM = quantitative susceptibility mapping; SN = substantia nigra; STN = subthalamic nucleus; T2w = spin-echo T2-weighted; WM (or wm) = white matter. SUBMITTED August 29, 2017. ACCEPTED March 1, 2018.

INCLUDE WHEN CITING Published online August 10, 2018; DOI: 10.3171/2018.3.JNS172145. 
tissue. ${ }^{43}$ These non-local blooming artifacts are removed in quantitative susceptibility mapping (QSM) ${ }^{14}$ a postprocessing technique employing deconvolution of GRE phase data for faithful depiction of tissue iron distribution..$^{24,44}$ QSM is regarded as the most promising technique for functional and stereotactic imaging of the STN. $3,11,13,25,37$

A detailed QSM data acquisition protocol has yet to be described for the DBS community. Here, we present a high-resolution QSM protocol for submillimeter imaging of deep gray matter structures that would improve identification and demarcation of STN subregions for DBS targeting.

\section{Methods}

Major parameters targeted in the protocol design include: submillimeter acquired voxel size, whole brain coverage, and clinically acceptable scan time (acquisition time less than 15 minutes). Since high-resolution MR data might suffer from noise, additional tests were carried out to test the performance of QSM with millimeter-scale voxel sizes.

\section{Imaging Protocol}

A 3-T MR system (General Electric Healthcare) with an 8-channel head coil was used to acquire all the MR images. Sagittal 3D T2w (CUBE) and axial 3D GRE imaging were performed. Detailed parameters are summarized in Table 1. Both GRE and CUBE sequences were used to acquire data from healthy volunteers. Only GRE sequences were used to acquire data from Parkinson's disease (PD) patients.

\section{Population Characteristics}

Seven healthy volunteers ( 7 men, age range $25-46$ years, mean age 31 years) were included in this study. Additionally, data from 10 randomly chosen PD patients (5 men, 5 women, age range 51-67 years, mean age 58 years) who underwent MRI for presurgical DBS planning from May 2016 to August 2016 were retrospectively analyzed. Indications for STN-DBS surgery included refractory motor fluctuations, medically refractory tremors, and drug-induced dyskinesias. This study was approved by the Weill Cornell IRB and the prospective subjects gave written consent.

\section{Image Analysis}

QSM was reconstructed from the acquired high-resolution GRE data at $0.5-\mathrm{mm}$ isotropic resolution. Then, additional data sets for each contrast were synthesized with thicker axial slices to simulate low-resolution acquisition by cropping in $\mathrm{k}$-space along the $\mathrm{k}_{\mathrm{z}}$ direction. The final axial slice thickness of these low-resolution data was set to $2 \mathrm{~mm}$ for QSM reconstruction. All resulting images (original and down-sampled) were then co-registered using FSL toolbox ${ }^{20}$ to high-resolution $\mathrm{T} 2 * \mathrm{w}$ images for further analyses.

\section{Qualitative Evaluation}

A radiologist (A.G., 11 years of experience), who was blinded to the resolution of acquired images, evaluated the appearance of the STN on T2w and QSM images from
TABLE 1. Imaging parameters

\begin{tabular}{lll}
\hline \multicolumn{1}{c}{ Parameter } & \multicolumn{1}{c}{ 3D T2w } & \multicolumn{1}{c}{ 3D GRE } \\
\hline Imaging plane & Sagittal & Axial \\
\hline TR $(\mathrm{msec})$ & 3000 & 44.1 \\
\hline TE $(\mathrm{msec})$ & 92.3 & $\begin{array}{c}\text { TE1 }=3.8, \Delta \mathrm{TE}=4.06, \\
\text { \#TE }=10\end{array}$ \\
\hline ETL & 100 & \multicolumn{1}{c}{-} \\
\hline Bandwidth $(\mathrm{kHz})$ & \pm 62.5 & \pm 62.5 \\
\hline NEX & 1 & 0.75 \\
\hline Acceleration factor & Slice $=2$ & ASSET = 2 \\
\hline FOV (cm) & 25.6 & 25.6 \\
\hline Phase FOV & 1 & 0.8 \\
\hline Acquisition matrix & $320 \times 320 \times 220$ & $320 \times 320 \times 180$ \\
\hline Voxel (mm $\left.{ }^{3}\right)$ & & \\
$\quad$ Acquired & $0.8 \times 0.8 \times 0.8$ & $0.8 \times 0.8 \times 0.8-1.0$ \\
$\quad$ Reconstructed & $0.5 \times 0.5 \times 0.5$ & $0.5 \times 0.5 \times 0.5$ \\
\hline Scan time & 13 mins & 13 mins \\
\hline
\end{tabular}

healthy volunteers and visually graded the overall image quality on a 5-point scale based on the demarcation of the STN margins and definition of the intensity gradient due to changes in iron concentration: grade 4, all STN borders are clearly defined, and the gradient along the STN is visible; grade 3, all STN borders are clearly defined, but the gradient is not visible; grade 2, one or more border(s) are poorly defined, gradient is clearly visible; grade 1 , one or more border(s) are poorly defined, gradient is not visible; grade 0, STN is not visible in the image.

\section{Quantitative Analysis}

STNs were identified by their signal intensities (hypointense on T2w images and hyperintense on QSM) and locations relative to the anterior commissure (AC)-posterior commissure (PC) line. Using a multimodal approach on the original high-resolution images, STNs were segmented manually in ITK-SNAP ${ }^{50}$ on 2 consecutive axial slices located approximately $3.2 \mathrm{~mm}$ below the AC-PC line. This segmentation (approximately $0.25 \mathrm{~cm}^{2}$ per STN on each slice) was further subdivided into 4 quadrants - anterolateral (AL), anteromedial (AM), posterolateral (PL), and posteromedial (PM) - based on main axes of the STN octagon in the axial plane (Fig. 1). To create ROIs (regions of interest) containing adjacent white matter, a $1.5-\mathrm{mm}-$ thick band immediately surrounding the STN was drawn. To characterize visibility of each STN quadrant on QSM and $\mathrm{T} 2 \mathrm{w}$ images, their contrast-to-noise ratios (CNRs) with respect to immediately adjacent white matter (WM) were calculated for each slice resolution according to the following definition:

$$
C N R_{W M}=\frac{\left|I_{j}-I_{w m_{j}}\right|}{\sigma_{w m_{j}}} .
$$

Here $I_{j}$ is the average intensity of one of the 4 STN quadrants, $I_{w m_{j}}$ is average WM intensity, and $\sigma_{w m_{j}}$ is the standard deviation of intensity within the closest to $I_{j}$ neighboring white matter sector. 


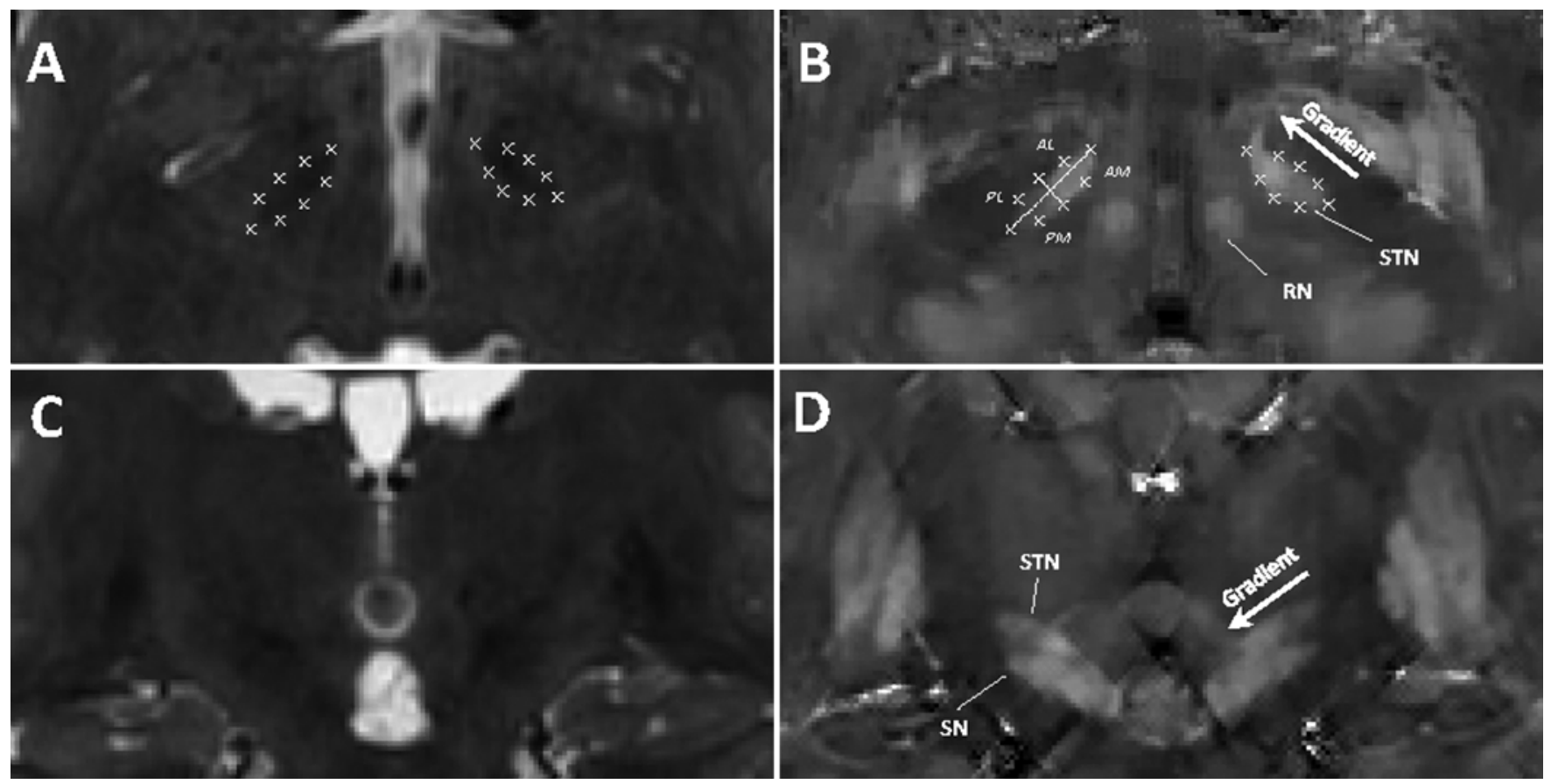

FIG. 1. Axial and coronal views of the STN in T2w (A and C) and QSM (B and D) images from a healthy 25-year-old man showing the subthalamic nucleus (STN), substantia nigra (SN), and red nucleus (RN). Border points indicated by " $x$ " were used to create bilateral masks of the nuclei $(A)$ and to define the 4 STN quadrants: anterolateral (AL), anteromedial (AM), posterolateral (PL), and posteromedial (PM) (B). STN susceptibility gradient (shown with arrows) is clearly visible in the QSM images.

To assess the sensitivity of each sequence to the heterogeneous distribution of iron within the STN, contrast ratios between adjacent quadrants were estimated. The contrast ratio between regions $i$ and $j$ was defined as $C R_{i j}$ $=\left(I_{i}-I_{j}\right) / I_{j}$.

\section{Statistical Analysis}

Statistical analysis was performed using MATLAB routines. The data were reported as mean $\pm \mathrm{SD}$. Comparisons of CNR values for each resolution were performed using the Wilcoxon signed-rank test. For visualization scores, the Wilcoxon rank-sum test was employed to test the difference between imaging methods for both resolutions.

\section{Results}

\section{Healthy Subject Study}

\section{Qualitative Results}

The STN was successfully imaged in all 7 volunteers. The average visualization scores for QSM and T2w images in high/low resolutions were 3.86/2.0 and 1.886/1.0, respectively. The difference between scores for high-resolution QSM and T2w images was significant $(\mathrm{p}<0.005)$, while no significant difference was observed in the lowresolution image sets. Switching to high resolution led to a significant increase in the radiologist's assessment of the QSM images $(\mathrm{p}<0.005)$, but not the T2w images. According to the scores, an intensity gradient was observed in 6 out of 7 high-resolution QSM cases and 1 out of 7 low-resolution cases. The gradient was not visible in any $\mathrm{T} 2 \mathrm{w}$ images.

\section{Quantitative Results}

The results of the CNR evaluation are summarized in Table 2. For 0.5-mm slice thickness, the CNRs of all STN quadrants on axial QSM images were higher than those of STN quadrants on axial T2w images ( $p<0.05)$. The CNR in QSM was increased compared to T2w by an average factor of $1.94 \pm 0.42$. For the 2 -mm slice resolution, a statistically significant increase in the CNR of QSM relative to that of $\mathrm{T} 2 \mathrm{w}$ was observed only in the lateral quadrants in both left and right STN. When comparing low and high resolutions, an increase in voxel size negatively affects the CNR in QSM $(\mathrm{p}<0.05)$ in all quadrants.

TABLE 2. Characterization of the STN quadrants contrast-tonoise ratios (CNR) relative to adjacent tissue

\begin{tabular}{|c|c|c|c|c|c|}
\hline \multirow[b]{2}{*}{ Quadrant } & \multirow[b]{2}{*}{ Image } & \multicolumn{2}{|c|}{ QSM } & \multicolumn{2}{|c|}{$\mathrm{T} 2 \mathrm{w}$} \\
\hline & & $0.5 \mathrm{~mm} \dagger$ & $2 \mathrm{~mm} \dagger$ & $0.5 \mathrm{~mm} \dagger$ & $2 \mathrm{~mm} \dagger$ \\
\hline \multirow{2}{*}{ Anterolateral } & Rt STN & $4.5 \pm 1.7$ & $4.0 \pm 1.3^{*}$ & $2.2 \pm 0.8$ & $1.7 \pm 0.6^{*}$ \\
\hline & Lt STN & $4.6 \pm 1.4$ & $4.0 \pm 1.1^{*}$ & $2.3 \pm 1.1$ & $1.8 \pm 0.9^{*}$ \\
\hline \multirow{2}{*}{ Anteromedial } & Rt STN & $4.9 \pm 0.8$ & $2.7 \pm 0.5^{*}$ & $3.4 \pm 1.3$ & $3.3 \pm 1.2$ \\
\hline & Lt STN & $5.0 \pm 0.8$ & $3.2 \pm 0.3^{*}$ & $3.6 \pm 2.0$ & $3.6 \pm 1.7$ \\
\hline \multirow{2}{*}{ Posterolateral } & Rt STN & $5.1 \pm 1.8$ & $3.9 \pm 1.0^{*}$ & $2.3 \pm 0.4$ & $2.2 \pm 0.4$ \\
\hline & Lt STN & $4.9 \pm 1.4$ & $4.1 \pm 1.2^{*}$ & $1.8 \pm 0.3$ & $1.7 \pm 0.3$ \\
\hline \multirow{2}{*}{ Posteromedial } & Rt STN & $3.7 \pm 0.6$ & $2.6 \pm 0.7^{*}$ & $1.9 \pm 1.1$ & $1.9 \pm 0.6$ \\
\hline & Lt STN & $4.1 \pm 1.7$ & $3.4 \pm 1.8^{*}$ & $2.3 \pm 0.8$ & $2.2 \pm 1.2$ \\
\hline
\end{tabular}

† Slice thickness.

* Statistically significant difference $(p<0.05)$ between resolutions. 

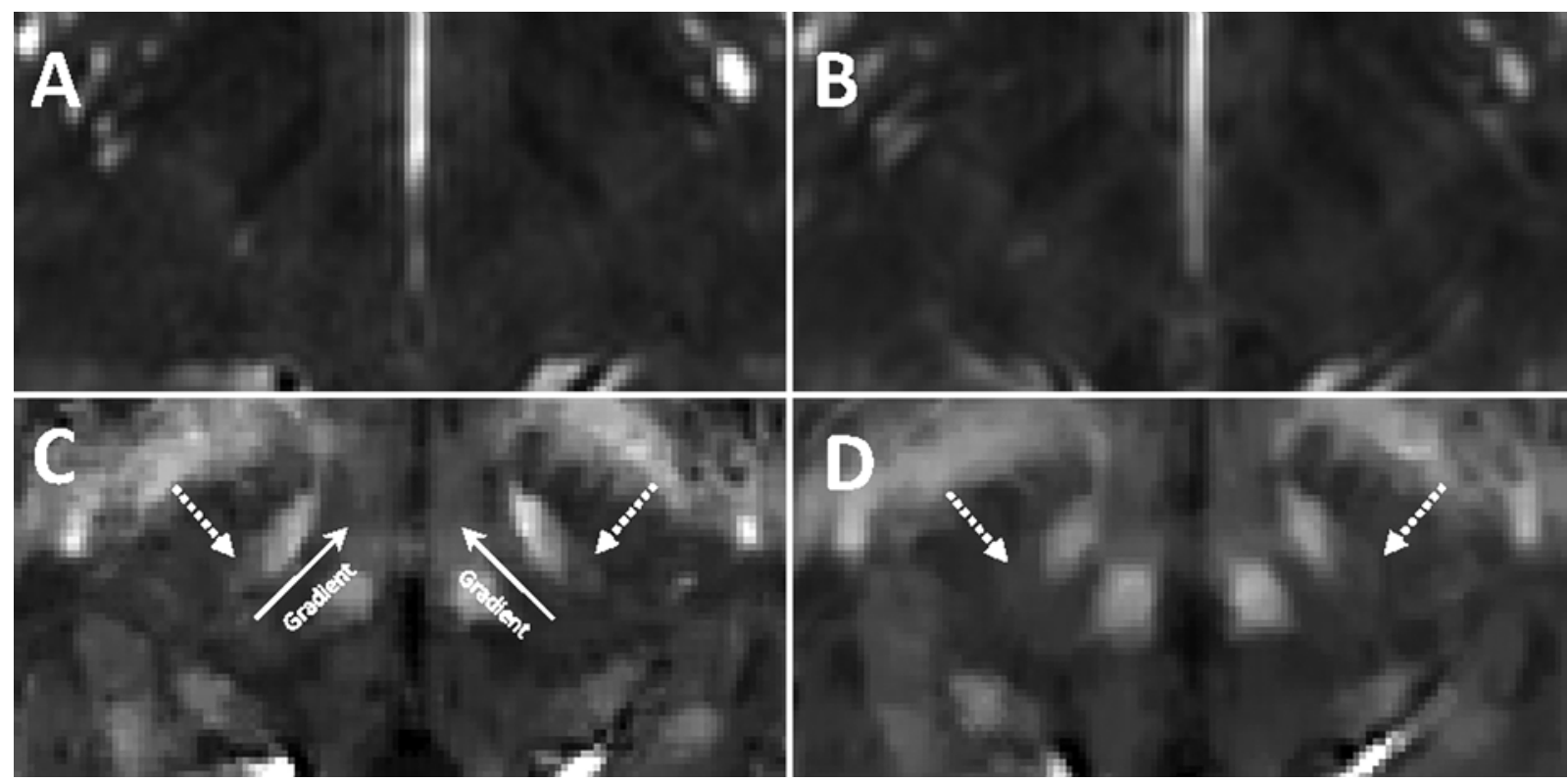

FIG. 2. Effects of slice thickness on STN-vs-tissue contrast in T2w and QSM images in a healthy 33-year-old man. A and C: T2w (A) and QSM (C) images with slice thickness of $0.5 \mathrm{~mm}$. B and D: T2w (B) and QSM images (D) with slice thickness of $2 \mathrm{~mm}$. Visibility of STN in T2w is relatively insensitive to slice thickness, as indicated by CNR measurements and the T2w image scores given by the radiologist. However, low resolution causes susceptibility underestimation in QSM, which led to significantly reduced contrast in the posterolateral quadrant (dashed arrows). Unlike T2w, QSM demonstrates a clearly visible gradient toward the caudal STN pole $(-0.024 \mathrm{ppm} / \mathrm{mm})$ in both hemispheres.

In T2w images, statistical significance was achieved only for anterolateral quadrants of the right STN. A representative comparison of the 2 different resolutions is shown in Fig. 2.

For both resolutions, QSM demonstrated a higher contrast ratio (CR) between the anterior and posterior quadrants than T2w for all considered pairs of STN quadrants (with the exception of right AL-AM) (Table 3). Figure 3 shows a comparison of intensity profiles extracted from representative T2w and QSM images obtained in one healthy volunteer along the axis connecting medial-inferior and posterior-lateral poles of the STN. Contrast between the anterior and posterior regions of the STN and a gradual decrease in the concentration of susceptibility sources are more obvious in the profile for the suscepti-

\section{TABLE 3. STN inter-quadrant contrast ratios}

\begin{tabular}{|c|c|c|c|c|c|}
\hline & \multirow[b]{2}{*}{ Image } & \multicolumn{2}{|c|}{ QSM } & \multicolumn{2}{|c|}{$\mathrm{T} 2 \mathrm{w}$} \\
\hline & & $0.5 \mathrm{~mm} \dagger$ & $2 \mathrm{~mm} \dagger$ & $0.5 \mathrm{~mm} \dagger$ & $2 \mathrm{~mm} \dagger$ \\
\hline PL to & Rt STN & $-0.7 \pm 0.6$ & $-0.7 \pm 0.7$ & $-0.0 \pm 0.1$ & $0.0 \pm 0.1$ \\
\hline PM & Lt STN & $-1.0 \pm 0.8$ & $-0.8 \pm 0.6$ & $0.0 \pm 0.1$ & $0.0 \pm 0.1$ \\
\hline PL to & Rt STN & $-0.8 \pm 0.3$ & $-0.8 \pm 0.3$ & $0.2 \pm 0.1$ & $0.2 \pm 0.1$ \\
\hline$A L$ & Lt STN & $-0.9 \pm 0.2$ & $-0.8 \pm 0.3$ & $0.2 \pm 0.1$ & $0.2 \pm 0.1$ \\
\hline AL to & Rt STN & $-0.2 \pm 0.1$ & $-0.2 \pm 0.2$ & $-0.2 \pm 0.1$ & $-0.1 \pm 0.1$ \\
\hline AM & Lt STN & $-0.2 \pm 0.04$ & $-0.2 \pm 0.1$ & $-0.02 \pm 0.1$ & $-0.1 \pm 0.1$ \\
\hline PM to & Rt STN & $-0.7 \pm 0.2$ & $-0.7 \pm 0.2$ & $0.1 \pm 0.1$ & $0.0 \pm 0.03$ \\
\hline AM & Lt STN & $-0.6 \pm 0.2$ & $-0.6 \pm 0.2$ & $0.1 \pm 0.1$ & $0.1 \pm 0.1$ \\
\hline
\end{tabular}

† Slice thickness. bility map than in T2w data (Fig. 3). STN sizes along its 3 main axes (mediodorsal-lateroventral, dorsolateral-ventromedial, and rostro-caudal) in QSM images were (3.2 \pm $0.3) \times(5.3 \pm 0.3) \times(11.8 \pm 0.9) \mathrm{mm}^{3}$ for the left and $(3.1$ $\pm 0.2) \times(5.3 \pm 0.4) \times(11.0 \pm 0.9) \mathrm{mm}^{3}$ for the right STN Similar measurements performed on T2w images resulted in the sizes of $(3.1 \pm 0.7) \times(5.8 \pm 0.7) \times(10.8 \pm 1.2) \mathrm{mm}^{3}$ for the left and $(3.2 \pm 0.4) \times(5.4 \pm 0.5) \times(10.5 \pm 1.1) \mathrm{mm}^{3}$ for the right STN. No statistically significant differences in sizes measured on both modalities were observed, although T2w-based measurements appear to have overall higher variation. The STN susceptibility gradient over all subjects was $-0.022 \pm 0.004 \mathrm{ppm} / \mathrm{mm}$.

\section{Patient Study}

QSM was successfully reconstructed in all 10 patient cases. Figure 4 shows QSM of the STN in the axial and coronal planes. In all cases, high contrast between STN and white matter and the ability to distinguish the STN from the substantia nigra (SN) were achieved (Table 4). The measured dimensions were $(3.2 \pm 0.2) \times(5.6 \pm 0.6)$ $\times(12.5 \pm 1.7) \mathrm{mm}^{3}$ for the left and $(3.1 \pm 0.2) \times(5.5 \pm$ $0.5) \times(11.8 \pm 1.8) \mathrm{mm}^{3}$ for the right nucleus. The average QSM STN gradients were $-0.026 \pm 0.009 \mathrm{ppm} / \mathrm{mm}$ and $-0.029 \pm 0.008 \mathrm{ppm} / \mathrm{mm}$ for the left and right nuclei, respectively.

\section{Discussion}

The results of the current study suggest that contrast in QSM is superior to that of T2w for delineation of the STN with respect to surrounding white matter. Submillimeter 
Dimov et al.
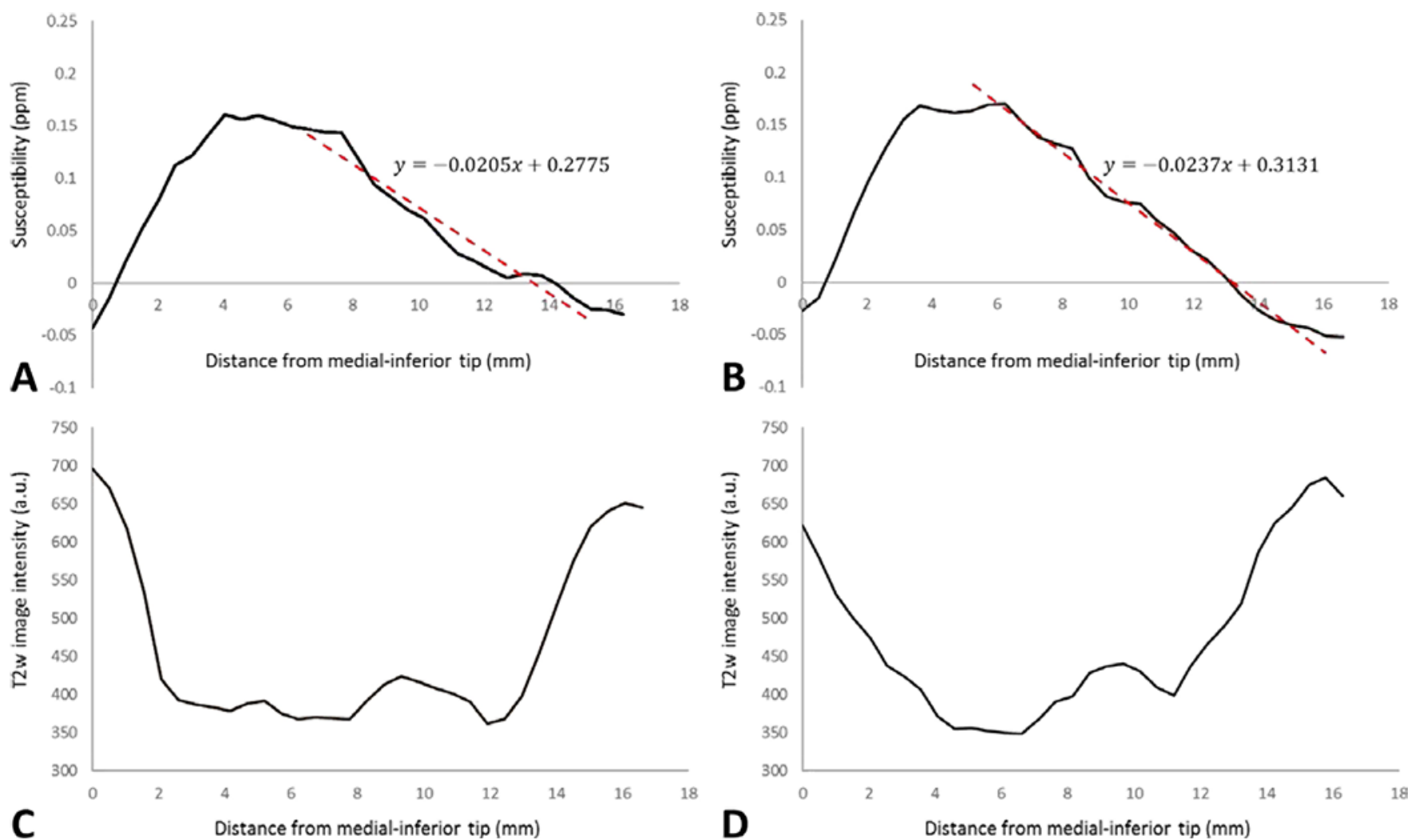

FIG. 3. Comparison of intensity profiles extracted from submillimeter-resolution QSM (A and B) and T2W (C and D) images of STN along the rostro-caudal axis. A and C: Left STN. B and D: Right STN. QSM consistently indicates a linear decrease in iron concentration (red dashed line), with an average susceptibility gradient of $-0.022 \mathrm{ppm} / \mathrm{mm}$. a.u. $=$ arbitrary units. Figure is available in color online only.

voxel size QSM faithfully depicts high iron content within the deep gray matter nuclei, allowing for confident localization of borders and observation of iron content het- erogeneity linked to functional subdivisions of the STN. High-resolution QSM may be used for precise neurosurgical targeting in planning DBS, such as STN and globus
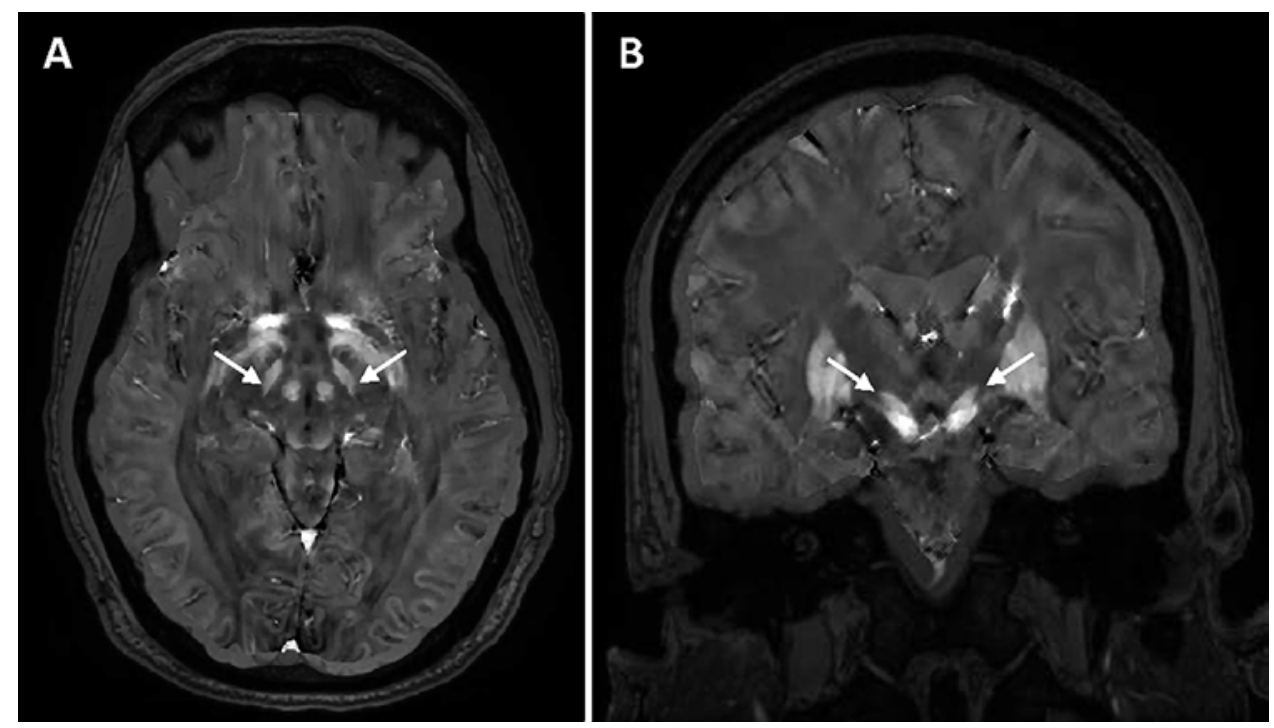

FIG. 4. QSM of STN (arrows) in axial (A) and coronal (B) planes in a 58-year-old man with PD. QSM provided good spatial definition of STN geometry; provided the ability to differentiate between STN, SN, and surrounding white matter; and visualized a decrease in iron toward the dorsolateral portion of the nucleus. 
TABLE 4. Characterization of the STN quadrant CNRs relative to adjacent tissue in healthy controls and PD patients

\begin{tabular}{cccc}
\hline \multirow{2}{*}{ Quadrant } & & \multicolumn{2}{c}{ QSM } \\
\cline { 3 - 4 } Anterolateral & Image & Healthy Controls & PD Patients \\
\cline { 2 - 4 } & Rt STN & $4.5 \pm 1.7$ & $4.8 \pm 0.9$ \\
\cline { 2 - 4 } Lt STN & $4.6 \pm 1.4$ & $4.2 \pm 1.0$ \\
\hline \multirow{2}{*}{ Anteromedial } & Rt STN & $4.9 \pm 0.8$ & $6.3 \pm 1.4^{*}$ \\
\cline { 2 - 4 } & Lt STN & $5.0 \pm 0.8$ & $5.5 \pm 2.0$ \\
\hline \multirow{2}{*}{ Posterolateral } & Rt STN & $5.1 \pm 1.8$ & $5.4 \pm 1.3$ \\
\cline { 2 - 4 } & Lt STN & $4.9 \pm 1.4$ & $5.5 \pm 1.7^{*}$ \\
\hline \multirow{2}{*}{ Posteromedial } & Rt STN & $3.7 \pm 0.6$ & $5.6 \pm 1.2^{*}$ \\
\cline { 2 - 4 } & Lt STN & $4.1 \pm 1.7$ & $5.4 \pm 1.6^{*}$ \\
\hline
\end{tabular}

${ }^{*}$ Statistically significant difference $(p<0.05)$ between cohorts.

pallidus internus (GPi) DBS for medically complicated Parkinson's disease (PD) and GPi DBS for dystonia.

Over the last decade, direct visualization of the STN for preoperative planning has been established using MRI to map anatomical details., ${ }^{4,17,38}$ High image quality is imperative to ensure efficacy of this patient-specific approach; given the variability between patients and complexity of the region, it is of great importance to have submillimeter resolution and high-contrast images that provide visibility of all parts of the STN. Traditional T2w MRI has been used in STN targeting for DBS. High iron content within the subthalamic nuclei shortens the transverse relaxation time, making the STN appear hypointense in these images. Recently, inconsistencies between the STN borders estimated on T2w images, histology, and intraoperative microelectrode recordings (MERs) have been reported in multiple studies..$^{12,16,18,33,39,42}$ One of the sources for these inconsistences is thought to be the inhomogeneous distribution of iron within the STN,,$^{13,16}$ with the lowest iron concentration typically observed in the dorsolateral region usually targeted in PD patients due to its correspondence to the sensorimotor area. ${ }^{34,36,41}$ This heterogeneous iron distribution in the STN is blurred on T2w MRI (Fig. 3). Fundamentally, T2 shortening is caused by field variance experienced by water protons during diffusion and chemical exchange, as known in the Solomon-Bloembergen-Morgan theory for relaxation enhancement by a paramagnetic substance $;^{8,40,43}$ the field is always blurred as a weighted sum of contributions from all surrounding iron. This blurring in T2w MRI explains the small improvement in STN contrast as T2w imaging resolution increases (Tables 2 and 3). The blurring in T2w MRI may be worsened in PD patients, who have elevated levels of iron in their deep gray matter nuclei. ${ }^{15}$

The STN is known to be involved in regulation of limbic, associative, and motor functions. ${ }^{19,22,46}$ Fibers from functionally distinct cortical areas converge in the STN according to the tripartite model: motor subdivision in the dorsolateral region, associative subdivision the central region, and the limbic subdivision in the medial tip, with functional overlap existing between these 3 subdivisions. ${ }^{5,19,30}$ Iron plays a significant role in regulation of glutamate production and secretion and is an important cofactor for the neurochemistry of these functional regions of the STN. ${ }^{1,29}$ Iron is heterogeneously distributed in the STN ${ }^{37}$ and a strong correlation between iron concentration measured by QSM and densities of axonal passage measured on diffusion tensor imaging has been reported. ${ }^{32}$ These understandings of STN function and anatomy are consistent with clinical evidence that the dorsolateral region of the STN-that appears to be less paramagnetic compared to the other STN regions - may be the optimal site for DBS targeting to avoid negative side effects while maximizing effects on the motor system..$^{2,9,48}$

QSM can be used to overcome inadequacies of relaxation-based contrasts in T2w MRI. ${ }^{25}$ Due to the sensitivity of QSM to the presence of biometals, this technique has been used extensively in studying the iron-rich structures of deep gray matter. Particularly, QSM has been successfully used in STN targeting, ${ }^{34}$ where its application resulted a single intraoperative MER in over $85 \%$ of cases. The comparative analyses of QSM and T2w presented here suggest that susceptibility mapping is better suited for high-resolution preoperative STN imaging. QSM can offer better visualization of iron distribution within the STN, providing identification of optimal DBS targeting sites. The qualitative and quantitative results also suggest that QSM-unlike T2w-benefits from high-resolution acquisition, which makes it valuable for accurate segmentation of the target areas without the need for image interpolation.

High-resolution QSM-based estimation of STN dimensions $\left(\sim 3 \times 5 \times 12 \mathrm{~mm}^{3}\right)$ agree with histological studies and microelectrode recordings. ${ }^{10,27,28,42,49}$ QSM with submillimeter resolution can provide adequate spatial resolution to guide the DBS electrode that is typically $1.3 \mathrm{~mm}$ in diameter and $1.5 \mathrm{~mm}$ in height. MER is arguably the reference standard for measuring STN dimensions in vivo, but it is invasive and may only provide 2-dimensional information, requiring multiple electrode insertions. In contrast, high-resolution QSM is noninvasive and provides direct 3D visualization of STN subdivisions.

In this study, the comparison between QSM and T2w was limited to healthy volunteers; it was not possible on patient data because T2w MRI had been discarded from the preoperative presurgical DBS imaging protocol. Furthermore, raw GRE data were not retained, making it impossible to compare the effects of change in image resolution. For large brain volume coverage in the patient study, increased slice thickness (to $1 \mathrm{~mm}$ ) at the same scan time was chosen; an alternative option would be to increase scan time while maintaining isotropic acquisition resolution.

In summary, high-resolution QSM provides better definition of the STN from surrounding tissue and between its subregions than traditional T2w MRI. Furthermore, submillimeter QSM of the STN may be beneficial in DBS targeting.

\section{Acknowledgments}

This investigation was supported in part by grants R01CA178007, R01CA178007, R21EB024366, R01NS090464, R01NS095562, R01NS095562, and S10OD021782 from the National Institutes of Health. 


\section{References}

1. Abbruzzese G, Cossu G, Balocco M, Marchese R, Murgia D, Melis M, et al: A pilot trial of deferiprone for neurodegeneration with brain iron accumulation. Haematologica 96:17081711,2011

2. Akram H, Sotiropoulos SN, Jbabdi S, Georgiev D, Mahlknecht P, Hyam J, et al: Subthalamic deep brain stimulation sweet spots and hyperdirect cortical connectivity in Parkinson's disease. Neuroimage 158:332-345, 2017

3. Alkemade A, de Hollander G, Keuken MC, Schäfer A, Ott DVM, Schwarz J, et al: Comparison of T2*-weighted and QSM contrasts in Parkinson's disease to visualize the STN with MRI. PLoS One 12:e0176130, 2017

4. Andrade-Souza YM, Schwalb JM, Hamani C, Hoque T, Saint-Cyr J, Lozano AM: Comparison of 2-dimensional magnetic resonance imaging and 3-planar reconstruction methods for targeting the subthalamic nucleus in Parkinson disease. Surg Neurol 63:357-363, 2005

5. Aravamuthan BR, Muthusamy KA, Stein JF, Aziz TZ, Johansen-Berg H: Topography of cortical and subcortical connections of the human pedunculopontine and subthalamic nuclei. Neuroimage 37:694-705, 2007

6. Bejjani BP, Dormont D, Pidoux B, Yelnik J, Damier P, Arnulf I, et al: Bilateral subthalamic stimulation for Parkinson's disease by using three-dimensional stereotactic magnetic resonance imaging and electrophysiological guidance. J Neurosurg 92:615-625, 2000

7. Benabid AL, Chabardes S, Mitrofanis J, Pollak P: Deep brain stimulation of the subthalamic nucleus for the treatment of Parkinson's disease. Lancet Neurol 8:67-81, 2009

8. Bloembergen N, Morgan LO: Proton relaxation times in paramagnetic solutions. Effects of electron spin relaxation. $\mathbf{J}$ Chem Phys 34:842-850, 1961

9. Bronstein JM, Tagliati M, Alterman RL, Lozano AM, Volkmann J, Stefani A, et al: Deep brain stimulation for Parkinson disease: an expert consensus and review of key issues. Arch Neurol 68:165, 2011

10. Bubnov AN: [Individual anatomic variability of the subthalamic nucleus in frontal sections.] Arkh Anat Gistol Embriol 68:85-87, 1975 (Russian)

11. Chandran AS, Bynevelt M, Lind CR: Magnetic resonance imaging of the subthalamic nucleus for deep brain stimulation. J Neurosurg 124:96-105, 2016

12. Cuny E, Guehl D, Burbaud P, Gross C, Dousset V, Rougier A: Lack of agreement between direct magnetic resonance imaging and statistical determination of a subthalamic target: the role of electrophysiological guidance. J Neurosurg 97:591-597, 2002

13. de Hollander G, Keuken MC, Bazin PL, Weiss M, Neumann J, Reimann K, et al: A gradual increase of iron toward the medial-inferior tip of the subthalamic nucleus. Hum Brain Mapp 35:4440-4449, 2014

14. de Rochefort L, Liu T, Kressler B, Liu J, Spincemaille P, Lebon V, et al: Quantitative susceptibility map reconstruction from MR phase data using bayesian regularization: validation and application to brain imaging. Magn Reson Med 63:194-206, 2010

15. Dexter DT, Carayon A, Javoy-Agid F, Agid Y, Wells FR, Daniel SE, et al: Alterations in the levels of iron, ferritin and other trace metals in Parkinson's disease and other neurodegenerative diseases affecting the basal ganglia. Brain 114:1953-1975, 1991

16. Dormont D, Ricciardi KG, Tandé D, Parain K, Menuel C, Galanaud D, et al: Is the subthalamic nucleus hypointense on T2-weighted images? A correlation study using MR imaging and stereotactic atlas data. AJNR Am J Neuroradiol 25:1516-1523, 2004

17. Dormont D, Seidenwurm D, Galanaud D, Cornu P, Yelnik J, Bardinet E: Neuroimaging and deep brain stimulation. AJNR Am J Neuroradiol 31:15-23, 2010
18. Hamani C, Richter EO, Andrade-Souza Y, Hutchison W, Saint-Cyr JA, Lozano AM: Correspondence of microelectrode mapping with magnetic resonance imaging for subthalamic nucleus procedures. Surg Neurol 63:249-253, 2005

19. Haynes WI, Haber SN: The organization of prefrontal-subthalamic inputs in primates provides an anatomical substrate for both functional specificity and integration: implications for basal ganglia models and deep brain stimulation. J Neurosci 33:4804-4814, 2013

20. Jenkinson M, Beckmann CF, Behrens TE, Woolrich MW, Smith SM: FSL. Neuroimage 62:782-790, 2012

21. Kerl HU, Gerigk L, Pechlivanis I, Al-Zghloul M, Groden C, Nölte I: The subthalamic nucleus at 3.0 Tesla: choice of optimal sequence and orientation for deep brain stimulation using a standard installation protocol: clinical article. J Neurosurg 117:1155-1165, 2012

22. Lambert C, Zrinzo L, Nagy Z, Lutti A, Hariz M, Foltynie $\mathrm{T}$, et al: Confirmation of functional zones within the human subthalamic nucleus: patterns of connectivity and subparcellation using diffusion weighted imaging. Neuroimage 60:83-94, 2012

23. Lemaire JJ, Coste J, Ouchchane L, Caire F, Nuti C, Derost P, et al: Brain mapping in stereotactic surgery: a brief overview from the probabilistic targeting to the patient-based anatomic mapping. Neuroimage 37 (Suppl 1):S109-S115, 2007

24. Li J, Chang S, Liu T, Wang Q, Cui D, Chen X, et al: Reducing the object orientation dependence of susceptibility effects in gradient echo MRI through quantitative susceptibility mapping. Magn Reson Med 68:1563-1569, 2012

25. Liu T, Eskreis-Winkler S, Schweitzer AD, Chen W, Kaplitt MG, Tsiouris AJ, et al: Improved subthalamic nucleus depiction with quantitative susceptibility mapping. Radiology 269:216-223, 2013

26. Longhi M, Ricciardi G, Tommasi G, Nicolato A, Foroni R, Bertolasi L, et al: The role of $3 \mathrm{~T}$ magnetic resonance imaging for targeting the human subthalamic nucleus in deep brain stimulation for Parkinson disease. J Neurol Surg A Cent Eur Neurosurg 76:181-189, 2015

27. Massey LA, Miranda MA, Zrinzo L, Al-Helli O, Parkes HG, Thornton JS, et al: High resolution MR anatomy of the subthalamic nucleus: imaging at $9.4 \mathrm{~T}$ with histological validation. Neuroimage 59:2035-2044, 2012

28. Mavridis I, Boviatsis E, Anagnostopoulou S: Anatomy of the human subthalamic nucleus: a combined morphometric study. Anat Res Int 2013:319710, 2013

29. McGahan MC, Harned J, Mukunnemkeril M, Goralska M, Fleisher L, Ferrell JB: Iron alters glutamate secretion by regulating cytosolic aconitase activity. Am J Physiol Cell Physiol 288:C1117-C1124, 2005

30. Nambu A, Tokuno H, Inase M, Takada M: Corticosubthalamic input zones from forelimb representations of the dorsal and ventral divisions of the premotor cortex in the macaque monkey: comparison with the input zones from the primary motor cortex and the supplementary motor area. Neurosci Lett 239:13-16, 1997

31. Obeso JA, Olanow CW, Rodriguez-Oroz MC, Krack P, Kumar R, Lang AE: Deep-brain stimulation of the subthalamic nucleus or the pars interna of the globus pallidus in Parkinson's disease. N Engl J Med 345:956-963, 2001

32. Patel W, Dimov A, Wang Y, Yao Y, Kopell BH, O'Halloran $\mathrm{R}$ : Is iron concentration linked to structural connectivity in the subthalamic nucleus? Implications for planning of deep brain stimulation, presented at the ISMRM 25th Annual Meeting \& Exhibition, Honolulu, 2017 (Electronic Poster \#5277) (https://www.ismrm.org/17/program_files/EP17.htm) [Accessed April 16, 2018]

33. Polanski WH, Martin KD, Engellandt K, von Kummer R, Klingelhoefer L, Fauser M, et al: Accuracy of subthalamic nucleus targeting by T2, FLAIR and SWI-3-Tesla MRI 
confirmed by microelectrode recordings. Acta Neurochir (Wien) 157:479-486, 2015

34. Rasouli J, Ramdhani R, Panov FE, Dimov A, Zhang Y, Cho $\mathrm{C}$, et al: Utilization of quantitative susceptibility mapping for direct targeting of the subthalamic nucleus during deep brain stimulation surgery. Oper Neurosurg (Hagerstown) 14:412-419, 2018

35. Richter EO, Hoque T, Halliday W, Lozano AM, Saint-Cyr JA: Determining the position and size of the subthalamic nucleus based on magnetic resonance imaging results in patients with advanced Parkinson disease. J Neurosurg 100:541-546, 2004

36. Rodriguez-Oroz MC, Rodriguez M, Guridi J, Mewes K, Chockkman V, Vitek J, et al: The subthalamic nucleus in Parkinson's disease: somatotopic organization and physiological characteristics. Brain 124:1777-1790, 2001

37. Schäfer A, Forstmann BU, Neumann J, Wharton S, Mietke A, Bowtell R, et al: Direct visualization of the subthalamic nucleus and its iron distribution using high-resolution susceptibility mapping. Hum Brain Mapp 33:2831-2842, 2012

38. Schlaier J, Schoedel P, Lange M, Winkler J, Warnat J, Dorenbeck U, et al: Reliability of atlas-derived coordinates in deep brain stimulation. Acta Neurochir (Wien) 147:1175-1180, 2005

39. Schlaier JR, Habermeyer C, Warnat J, Lange M, Janzen A, Hochreiter A, et al: Discrepancies between the MRI- and the electrophysiologically defined subthalamic nucleus. Acta Neurochir (Wien) 153:2307-2318, 2011

40. Solomon I: Relaxation processes in a system of two spins. Phys Rev 99:559-565, 1955

41. Theodosopoulos PV, Marks WJ Jr, Christine C, Starr PA: Locations of movement-related cells in the human subthalamic nucleus in Parkinson's disease. Mov Disord 18:791-798, 2003

42. Verhagen R, Schuurman PR, van den Munckhof P, Contarino MF, de Bie RMA, Bour LJ: Comparative study of microelectrode recording-based STN location and MRI-based STN location in low to ultra-high field (7.0 T) T2-weighted MRI images. J Neural Eng 13:066009, 2016

43. Wang Y: Principles of Magnetic Resonance Imaging: Physics Concepts, Pulse Sequences, \& Biomedical Applications. Ithaca: Yi Wang, 2012

44. Wang Y, Liu T: Quantitative susceptibility mapping (QSM): Decoding MRI data for a tissue magnetic biomarker. Magn Reson Med 73:82-101, 2015
45. Wang Y, Spincemaille P, Liu Z, Dimov A, Deh K, Li J, et al: Clinical quantitative susceptibility mapping (QSM): Biometal imaging and its emerging roles in patient care. J Magn Reson Imaging 46:951-971, 2017

46. Wichmann T, Bergman H, DeLong MR: The primate subthalamic nucleus. I. Functional properties in intact animals. J Neurophysiol 72:494-506, 1994

47. Wichmann T, Delong MR: Deep brain stimulation for neurologic and neuropsychiatric disorders. Neuron 52:197-204, 2006

48. Wodarg F, Herzog J, Reese R, Falk D, Pinsker MO, Steigerwald F, et al: Stimulation site within the MRI-defined STN predicts postoperative motor outcome. Mov Disord 27:874879, 2012

49. Yelnik J, Percheron G: Subthalamic neurons in primates: a quantitative and comparative analysis. Neuroscience 4:17171743,1979

50. Yushkevich PA, Piven J, Hazlett HC, Smith RG, Ho S, Gee JC, et al: User-guided 3D active contour segmentation of anatomical structures: significantly improved efficiency and reliability. Neuroimage 31:1116-1128, 2006

\section{Disclosures}

Dr. Wang owns equity in Medimagemetric LLC, a Cornell spinoff company. Dr. Kopell is a consultant for Medtronic and Abbott Neuromodulation.

\section{Author Contributions}

Conception and design: Wang, Dimov. Acquisition of data: Dimov, Gupta, Kopell. Analysis and interpretation of data: Dimov. Drafting the article: Wang, Dimov, Kopell. Critically revising the article: all authors. Reviewed submitted version of manuscript: all authors. Statistical analysis: Dimov. Study supervision: Wang, Kopell.

\section{Correspondence}

Yi Wang: Cornell University, New York, NY. yiwang@med. cornell.edu. 